This item is the archived peer-reviewed author-version of:

\title{
Risk-informed decision making of safety investments by using the disproportion
} factor

\section{Reference:}

Talarico Luca, Reniers Genserik.- Risk-informed decision making of safety investments by using the disproportion factor

Process safety and environmental protection / Institution of Chemical Engineers [London] - ISSN 0957-5820 - 100(2016), p. 117130

Full text (Publisher's DOI): http://dx.doi.org/doi:10.1016/J.PSEP.2016.01.003

To cite this reference: http://hdl.handle.net/10067/1312130151162165141 


\title{
Risk-informed decision making of safety investments by using the disproportion factor
}

\author{
Luca Talarico ${ }^{\mathrm{a}}$, Genserik Reniers ${ }^{\mathrm{a}, \mathrm{b}, \mathrm{c}}$ \\ ${ }^{a}$ Faculty of Applied Economics, Research Groups ANT/OR, University of Antwerp, Prinsstraat 13, 2000 \\ Antwerp, Belgium \\ ${ }^{b}$ Safety Science Group, TU Delft, Jaffalaan 5, 2628 BX Delft, The Netherlands \\ ${ }^{c}$ Center for Corporate Sustainability (CEDON), HUB, KULeuven, Stormstraat 2, 1000 Brussels, Belgium
}

\begin{abstract}
To improve risk informed decision making, a systematic approach is developed. The goal is to analyze and to evaluate safety investments aimed at mitigating and preventing major accidents involving e.g. hazardous materials that might trigger significant financial losses and fatalities. A formulation, explicitly using a disproportion factor, is proposed as a simulation exercise approach to this end. The disproportion factor can be used by any private and public investor to bias decision-makers towards safety. This is especially interesting for deciding about the prevention of High Impact Low Probability (HILP) accidents.

Furthermore experimental simulations have been performed on realistic data to test the proposed decision model and to provide general recommendations. Several types of accidents were considered and the impacts of technical and financial parameters on the disproportion factor, possibly making a safety investment profitable from an economic perspective, are also investigated and discussed in this paper.
\end{abstract}

Keywords: Disproportion factor, Safety investment, Risk analysis, Decision model, Risk management.

\section{Introduction}

The concept of operational safety represents a top priority for many industries, and especially for those in the chemical sector, where due to the storage and processing of dangerous materials, major accidents may trigger significant losses, including damages to goods, harm to employees and even detriment to the surrounding communities [13].

For this reason, substantial resources are invested every year by public and private organizations to better understand the dangers associated with products/processes and to establish effective safety measures to protect people and the environment of chemical industrial areas. Depending on local circumstances, these measures are taken within a

Email addresses: luca.talarico@uantwerpen. be (Luca Talarico), genserik .reniers@uantwerpen.be (Genserik Reniers) 
comprehensive regulatory framework, designed at a national and/or international level. The approaches used to decide on which measures to take, are continuously reviewed to increase their effectiveness and at the same time to decrease cost, if possible.

From a single company point of view, safety investments, just like any other type of investment, might be economically profitable or not, depending on some key factors, as well as on how their financial effects are measured.

Several studies in the literature show that safety investments are not always financially beneficial if compared to the expected consequences of an accident [20]. In general, "soft" interventions having lower costs (e.g. training, simple equipment, small changes to work organization) tend to be more profitable than other expensive and more complex measures such as those involving equipment, infrastructure and so on [27].

Since public or private organizations need to justify the budget devoted to investments in safety, executive decision-makers usually assess, in a preliminary stage, the impact of safety measures addressing the following strategic and tactical questions: (a) how much should be spent on safety; (b) how much is the lack of safety costing to the business; and (c) what are the most cost-effective solutions[7].

In the UK, safety is prescribed for any public or private organization, so far as is "reasonably practicable". Assuming that safety measures are implementable from a technical point of view, the risk that is potentially faced without safety investment (the "do-nothing scenario") needs to be placed in one scale and the sacrifice, whether in money, time or trouble, involved in the measures necessary to avert the risk needs to be placed in the other. Whether there exists a significant disproportion between risk and sacrifice, a preliminary computation needs to be carried out in order to determine the boundaries, until the point at which the sacrifice can be considered to be reasonably practicable [2, 21].

In other words, a quantitative risk assessment is required to determine how much potential loss could be saved by investments in safety measures. Small and medium enterprises might face particular difficulties since they have limited access to capital. As a result, this inevitably often restricts safety-related investments to only those essential for company survival, meaning that long-term investments are a low priority, even if profitable [6]. Moreover, due to a potential lack of risk expertise, small sized companies can make erroneous safety decisions relying on the believe that their risks as well as the potential costs of accidents are also small, although incident rates for such firms in general are higher [9].

No matter the size of the business, safety related decisions always present economic implications. However, the way in which safety interventions are economically evaluated represents a critical issue, as many factors are difficult to measure. Moreover, organizations often have difficulties to accurately measure the effectiveness and the cost of related safety activities, since safety is usually an investment that provides hypothetical profit, namely the benefit due to the avoidance of accidents in some distant future. In general, assessments concerning safety investments are done ex ante mainly using a quantitative approach and including an evaluation of several aspects such as the legal framework, policies, impact and likelihood of potential accidents, safety budget etc. Schneider [23] highlighted that safety-related investments are rarely evaluated in economic terms. For instance, Smallman and John [25] published a survey in which it emerges that only a minority of British companies adopt a quantitative and 
well-structured approach to assess safety investments.

In the last years some studies addressed the problem of finding how much an organization should invest in safety. According to Gordon and Loeb [12] an asset of greater value should not necessarily benefit from a greater investment to protect it. Moreover, there is a point at which the marginal benefits from safety investments are negative. Other studies [33] demonstrated that there does not exist a fixed percentage for optimal safety investments, since this value might depend on a series of factors such as the assumptions which are used, the business sector, the methodology used to estimate it, etc. In many cases of major accidents solely costs seem to outweigh the hypothetical benefits and therefore to take reputation benefits, legislation repercussion benefits and other factors which are very difficult to measure, a disproportion factor (DF) needs to be used as a bias in favor of safety.

Although the importance of the DF to evaluate safety investments has been already recognized in several reports, official documents and publications [3, 10, 14, 30], a structured methodology to determine a value for the DF to be applied to specific situations is still missing and only few publications have covered this issue. In addition, no universal accounting model has prevailed as a standard method to be adopted and applied to analyze safety investment options.

The study proposed in this paper attempts to fill the existing gap in the scientific literature by proposing a high level decision model that takes into account both the main financial and economics elements related to the investment decision and some technical parameters arising from a risk assessment analysis.

Focusing on high consequence accidents characterized by a low probability of occurrence, a cost/benefit methodology based on the well-known Net Present Value (NPV) calculation is developed by explicitly considering the DF. The latter is used to emphasize the importance of safety over costs to prevent or mitigate accidents. The value of the $\mathrm{DF}$, for which the net present value associated to the safety investment is equal to zero, is used within a more robust economic safety assessment approach to support decision-makers ranking and classifying safety investments. As a result, the methodology could provide recommendations to decision-makers improving their capability to compare alternative safety investments for major accidents. The goal of the model is thus to provide a high level framework supporting the comparison of alternative investment options rather than defining ideal level of DF which may vary depending on different factors (e.g. industry, materials, dimension of the firm).

Based on this formulation, a simulation is carried out to obtain comparable and reliable evaluations while assessing safety investments, as well as exploring the relationships between the main financial, technical and economic variables. Using the results of this sensitivity analysis, private investors and/or policy makers can be informed by a numerical tool in their efforts towards making employees, citizens, infrastructure and systems more safe from major accidents.

To simplify the reading, some abbreviations and acronyms are used in the remainder of the paper, as summarized in Table 1

The paper is organized as follows. Section 2 presents a brief overview of the literature on how cost/benefit methodologies can be applied to evaluate safety investments. Section 3 describes an innovative approach which combines the NPV calculation with the DF to assess safety investments. Section 4 illustrates a high level decision-making 


\begin{tabular}{llll}
\hline Acronym & Description & Acronym & Description \\
\hline ALARP & As Low As Reasonably Practicable & IRR & Internal Rate of Return \\
ALE & Annual Loss Expectancy & MJS & Maximum Justifiable Spend \\
ARO & Annual Rate of Occurrence & NPV & Net Present Value \\
CBA & Cost Benefit Analysis & PF & Proportion Factor \\
CCPS & Center for Chemical Process Safety & QRA & Quantitative Risk Assessment \\
DF & Disproportion Factor & ROSI & Return on Security/Safety Investment \\
ENISA & European Network and Information Se- & SLE & Single Loss Expectancy \\
& curity Agency & & \\
EV & Expectation Value & TRA & Threat and Risk Assessment \\
FR & Failure Rate & VSL & Value of a Statistical Life \\
HILP & High Impact Low Probability & & \\
\hline
\end{tabular}

Table 1: List of acronyms used in the paper

process that can be used by decision-makers to compare and assess safety investments. In Section 5 the results of a simulation are explored in which several accident scenarios are tested and the relationships between the DF and the main technical and financial parameters associated to a set of safety measures leading to a NPV of zero, are investigated. Finally, Section 6 concludes the paper providing some recommendations and suggestions for future work.

\section{Literature review}

As mentioned before, safety investments are rarely perceived to be profitable from the point of view of a single firm [22, 29]. Since decisions related to safety are taken at a company level, decision-makers within companies should be provided with the best information related to the cost-effectiveness of each safety investment [31]. Safety investments can be treated as regular investments subject to quantitative methodologies to determine their economic desirability. However, it should be noticed that the scientific literature on business case studies for safety interventions is limited. The economic profitability of a safety-related intervention can be assessed borrowing some of the methodologies that are widely used to assess the financial impact of other general types of investments.

Andreoni [1] proposed a theoretical cost/benefit model in which the total cost associated to a specific level of safety within a company is compared with the consequences of accidents. This safety cost increases as the desired overall safety level is higher and thus the consequences of the accident are reduced. Conversely, lower safety investments lead to higher real costs due to the consequences of accidents. Both safety and accidents cost factors are compared on the same monetary basis to determine a curve for total costs which aggregate both prevention costs and consequences of accidents. According to this model a safety investment is worth undertaking when it leads to a lower total cost. The economically optimal level associated to safety measures is the one where the aggregate total cost is minimum.

Despite this model represents one of the first attempts to determine the optimal level of safety investments by following a quantitative approach, it relies on oversimplified assumptions. Miller et al. [18] and Mossink [19] criticized such model since, 
even if a safety level can be defined, it cannot be seen as a function of preventive safety investments alone. Moreover, since different safety measures present different levels of efficiency, a unequivocal relationship between a "level of safety" and safety investments is not realistic.

Risks involve too much variability and complexity to allow organizations to be ranged on a one-dimensional safety scale. Moreover, it is not easy to show a causal and quantifiable relationship between safety investments and improvements in the overall safety levels [17, 24].

Furthermore, such approach cannot be used for major accidents since it is impossible to assess the real cost of the resulting disasters since such major accidents are extremely rare. In addition, a satisfactory record of accident cases is not available and cannot be built up.

In order to assess the desired level of safety investments, an evaluation of how much potential loss is expected to be saved by an investment needs to be performed. However, estimating the amount of money possibly saved from losses is a hard task which, in the real world, requires more than straightforward application of simple formulas. Nevertheless, reasonable approximations are needed to serve the user's interest to compare alternative safety investments or to justify a decision.

Some key factors identified for an accurate quantitative risk assessment (QRA) aimed at evaluating the economic impact of a safety investment imply an evaluation of the following items: (a) Benefits and costs associated with the safety investment should be identified and quantified; (b) Inflation and reference period must be taken into account. In fact, consequences may occur over a long period after the safety investment decisions.

The classical financial approaches (e.g. calculating the return on investment) are not particularly appropriate to deal with safety-related investments, since such type of investments do not produce direct benefits, but rather they help avoiding potential losses due to accidents. Therefore, the monetary value of the investment is compared with the monetary value of the risk reduction.

Some of the existing economic approaches make use of indicators such as: (a) Payback Period (which measures the period of time required to reach the break-even point to fully recover the investment); (b) Net Present Value (the sum of the present values of benefit and cost cash flows over a time horizon); (c) Internal Rate of Return (which measures the rate of return making the net present value of all positive and negative cash flows from a particular investment equal to zero); (d) Profitability Index (ratio used to rank projects by quantifying the amount of value created per unit of investment); (e) Benefit/Cost Ratio (ratio between all benefits of a project and its costs. All benefits and costs are given in monetary terms and are expressed in discounted present values).

Additional methods are based on other measures such as the Annual Loss Expectancy (ALE), the Single Loss Expectancy (SLE), Return on Security/Safety Investment (ROSI), the Annual Rate of Occurrence (ARO) the Threat and Risk Assessment (TRA). For a more detailed study the reader is referred to Dorman [6], Flores et al. [8] and ENISA [7].

The approaches based on the Net Present Value (NPV) have proven to be the most common method to evaluate investments, due to its robustness, its level of detail and 
its potential to represent realistic scenarios. For this reason, in the remainder of the paper we have chosen to use this approach and to adapt it to the assessment of safety investments related to major accident prevention.

In this paper, we indeed refer to high consequences, low probability accidents for which relatively costly investments in safety might be required. As mentioned in [7] the key factors that should be addressed for this kind of safety-related decisions refer to: (a) the type of safety measures; (b) the type of accidents targeted; (c) the size of investment and; (d) the assessment methodology to be used.

When talking about high consequences, low probability accident scenarios, the term ALARP (As Low As Reasonably Practicable) is often used to identify a reasonable level of investments in safety [15]. More specifically, ALARP questions whether safety investments are situated within reasonable boundaries obtained by weighing the risk against the sacrifice needed to further reduce it.

In some cases, if the costs are clearly very high and the reduction in risk is only marginal, then it is likely that the situation is already ALARP and further improvements are not required. In other circumstances the improvements may be relatively simple or cheap to implement and the risk reduction significant: in such case the existing situation is unlikely to be ALARP and an improvement is required. But there are some instances where the situation is less clear-cut. In such cases, a more detailed comparison has to be undertaken. In these instances, a more formal Cost Benefit Analysis (CBA) may provide additional insight.

In general, safety decisions should be weighted in favor of health and safety in case of HILP (High Impact Low Probability) risks. Nevertheless, the process is not one of balancing the costs and benefits of measures but, rather, of adopting measures except where they are ruled out because they involve grossly disproportionate sacrifices. Since several factors come into play, there is no simple formula for computing what is "ALARP". However, safety-related decisions still need to be justified based on some form of CBA.

Moreover, when comparing the sacrifice (cost) and the risk reduction (benefits), the usual rule applied by a CBA model is that the measure should be adopted only if benefits outweigh costs. However, in ALARP decisions, the rule is that the measure must be adopted unless the sacrifice is grossly disproportionate to the risk. In this way, the costs can outweigh benefits and the measure could still be reasonably practicable to introduce. How much costs can outweigh benefits before being judged grossly disproportionate depends on factors which are summarized by the DF

In Thomas and Jones [28] the concept of DF is replaced by the "proportionality factor" and used to determine the Maximum Justifiable Spend (MJS) defined as follows:

$$
M J S=\text { cost of failure } \times \text { probability of failure } \times \text { proportionality factor }
$$

MJS is the amount of money it is worth spending, based on the magnitude of the risk, in order to reduce the risk to acceptable levels. It gives a criterion for identifying what additional risk reduction measures are worth considering. The author suggests that the measures that cost more than the MJS are to be discarded immediately. Those that are lower than the MJS should be implemented in order to reduce the risk and to achieve ALARP. There also exists a grey area where additional assessment efforts 
are required. The formula represents an alternative to the NPV within a CBA. In fact, it can be used to rank and classify alternative safety measures although it relies on preliminary computations and risk assessments to determine a reasonable level of the proportionality factor.

In the next sections more details are presented about how the NPV is to be utilized to evaluate safety investments. In addition the concept of disproportion factor is explained and used to influence decisions.

\section{Investment assessment using a quantitative method}

In this section an innovative quantitative and structured methodology to assess and analyze safety investments is presented. The goal is to provide an economics-based method which can act as a financial "eye-opener" for decision-makers, raising awareness of the costs of not investing in safety and addressing the need of ALARP safety measures. In summary, the approach we developed should serve as a measure for more risk-informed decision making. After having briefly introduced the concepts of the NPV and the DF a method integrating both measures, is presented.

\subsection{Overview of the net present value calculation}

In order to decide whether to accept or to reject an investment, the majority of organizations generally follows a structured cost-benefit analysis aimed at: (1) identifying potential costs and benefits; (2) calculating the present values of all costs and benefits; (3) comparing the total present value of costs and total present value of benefits.

In order to compare the total costs and the total benefits, made by costs and benefits occurring at different points in time, one needs to take a discount rate into account in the calculation to obtain their present values. Thus, during a cost-benefit analysis, all cash flows, from both costs and benefits in the future, need to be converted to values in the present. This conversion is carried out by discounting the cash flows by a discount rate. The latter represents the rate at which we are willing to give up consumption in the present in exchange for additional consumption in the future.

This is the basis of the net present value methodology which requires that all positive and negative contributions (or cash flows) due to an investment are evaluated on the same financial basis. In other words, all measures involved in the decision need to be expressed in a monetary form.

The well-known formula for the NPV is shown in Eq. (2) where $R_{t}$ represents the net (positive or negative) flow in year $t$. This value is obtained as the difference between the benefits and the costs associated to the safety measure in a specific year. The discount factor also known as opportunity cost of capital (i.e. the rate of return that could be earned on an investment in the financial markets with similar risk) is represented by the letter $i$.

$$
\mathrm{NPV}=\sum_{t=0}^{n} \frac{R_{t}}{(1+i)^{t}}=\sum_{t=0}^{n} \frac{B_{t}-C_{t}}{(1+i)^{t}}
$$


The terms $B_{t}$ and $C_{t}$ represent respectively the benefits (positive cash flow) and the costs (negative cash flow) achieved/incurred in time $t$. The reader is referred to Campbell and Brown [4] for more details.

An investment project is recommended when the total NPV of all cash flows is positive, and an investment project is usually rejected when the NPV is negative. Applied to operational safety, the NPV of a project expresses the difference between the total discounted present value of the hypothetical benefits and the total discounted present value of the safety costs. A positive NPV for a given safety investment indicates that the project benefits are larger than its costs.

It is evident that the cash flows, due to prevention costs and certainly hypothetical benefits, may be uncertain. Different approaches can be used in this regard. The cash flows can for example be expressed as expected values, taking the uncertainties in the form of probabilities into consideration, and also increasing the discount rate to outweigh the possibilities for unfavorable outcomes. It is also possible to take into account uncertainties by using scenario analyses and thus estimating cash flows for different scenario cases (e.g. worst-case and most credible case) and adopting a disproportion factor as done in the remainder of the paper.

\subsection{The concept of the disproportion factor}

In the literature, the disproportion factor is sometimes referred to as the gross disproportion to reflect an intended bias in favor of safety. In the remainder of the paper, without loss of generality we will consistently use the term disproportion factor with the same meaning of the gross disproportion factor to avoid confusion and for the sake of legibility. One of the first attempts to establish a procedural manner to derive a value for the disproportion factor associated to specific risk reduction measures is due to Goose [11].

The so-called proportion factor $(\mathrm{PF})$ indicates the ratio of the costs to the benefits. The PF can then be compared with the numerical value of the disproportion factor (DF) in order to determine whether the risk reduction measure is "grossly disproportionate" or not. More specifically, there is disproportion, when the costs of a potential safety measure grossly exceeds the value of the safety benefits obtained when the measure is implemented, as in Eq. 33:

$$
P F=\frac{\text { Costs }}{\text { Benefits }}>D F
$$

Concerning the range of values that this factor can take, DF is necessarily greater than 1 because otherwise it would mean a bias against safety (or in favor of "unsafety"). According to Goose [11], the value of the DF is rarely over 10 and should never be higher than 30 .

Theoretically, the DF is higher when the risk increases and is infinite when the risk level reaches the intolerable region (situated higher than the ALARP region), meaning, in fact, that the risk must be reduced no matter the cost. A scheme of calculation has been suggested by Goose [11], using three terms, called the "how factors" referred to as "how bad", "how risk" and "how variable". These factors are computed starting from the following values: (a) The sum of the failure rates, expressed in events per 
year $\left(\sum F R\right)$. (b) The expectation value $(E V)$ which is sometimes also called Potential Loss of Life and represents the average number of casualties expected per year. (c) The maximum potential fatalities $\left(N_{M A X}\right)$, which are the worst case scenario concerning the number of fatalities for a single event. (d) The ratio of $E V$ to $\sum F R$, representing the average number of fatalities per event $\left(N_{a v}\right)$.

There are different calculation models among which Goose [11] suggests to calculate the three "how" factors as follows:

The "how bad" factor represents the effect of $N_{a v}=\frac{E V}{\sum F R}$, the average number of fatalities per event, on the DF:

$$
\text { How bad }=\log _{10}(N a v)
$$

The "how risky" factor represents the effect of $E V$, the expectation value, on the DF:

$$
\text { How risky }=\log _{10}\left(10^{5} \cdot E V\right)
$$

The "how variable" factor represents the effect of the ratio $\frac{N_{\max }}{N a v}$ on the DF:

$$
\text { How variable }=\log _{10}\left(\frac{N_{\max }}{N a v}\right)
$$

The DF is then computed by summing the "how" factors and adding 3 (dimensionless) to this product:

$$
D F=\log (N a v)+\log \left(10^{5} \cdot E V\right)+\log \left(\frac{N_{\max }}{N a v}\right)+3
$$

In the following, we present a formula to derive the value of the DF which makes the net present value of a safety investment equal to zero. Using the results of such simulation exercise it is possible to compare alternative safety measures. Moreover, given the limitations and restricted understanding of the 3 how factors it is important to judge whether the DF associated to each safety measure "behaves" in a reasonable way.

\subsection{Quantitative assessment by using the $D F$} ble 2 .

With every safety investment three main features are associated, as shown in Ta-

\begin{tabular}{ll}
\hline Symbol & Description \\
\hline$e$ & $\begin{array}{l}\text { Effectiveness of the safety investment expressed as a per- } \\
\text { centage }\end{array}$ \\
$M$ & $\begin{array}{l}\text { Initial cost of the safety investment including the instal- } \\
\text { lation expressed in a specific currency } \\
\end{array}$ \\
& $\begin{array}{l}\text { Yearly recurring cost expressed as a percentage of the ini- } \\
\text { tial investment's cost } M\end{array}$ \\
\hline
\end{tabular}

Table 2: Features associated to a safety investment to be evaluated 
The cost of a safety investment can be divided into $M$, corresponding to the initial investments (e.g. purchasing cost of new equipment and materials directly related to the intervention ${ }^{\dagger}$ ) and $m$, the yearly recurring costs due to maintenance, energy costs, yearly equipment, depreciation and interest expenses, material, training costs, etc.

The safety investment is evaluated considering a time horizon $n$ that should be defined by the investor. More specifically the time horizon should be compatible with the asset life of the safety measure to be analyzed. In other words, within $n$ years, the safety investment is supposed to maintain its effectiveness, without any significant deterioration of its performance.

In order to assess the financial impact of a safety investment, Eq. (2) is adapted to the evaluation of the cost/effectiveness of a safety measure by explicitly including the disproportion factor $D F$. More specifically, the investment is represented by the cost of the safety measure to be evaluated $(M)$. This cost is supposed to be entirely sustained in the initial year (year 0) when the investment needs to be evaluated. Due to the characteristics of the measure, yearly recurring costs (due e.g. to maintenance activities) might be required over the time horizon in which the investment is evaluated. These recurring costs are needed to maintain the functionality of a safety measure and keep its effectiveness at its initial level. These costs are expressed as a percentage of the measure's initial cost and assumed to be sustained starting from year 1 until the time horizon $n$ in which the investment is evaluated. Therefore, the cost value $C_{t}$ to be considered in the formula of the NPV assumes the form shown in Eq. (8).

$$
C_{t}= \begin{cases}M, & \text { if } t=0 \\ M \cdot m, & \text { otherwise }\end{cases}
$$

On the other side, the hypothetical benefits are quantified as the monetary savings which can be achieved if the disruptions caused by an accident, that might happen with probability $p$, are avoided or mitigated thanks to the safety investment which has been pursued

To quantify the savings, the basic notion of risk is used. As defined by CCPS (Center for Chemical Process Safety) [5], risk can be seen as an index of potential economic loss, human injury, or environmental damage, that is measured in terms of both the incident probability and the magnitude of the loss, injury, or damage. The risk associated with a specific (unwanted) event can be expressed as the product of two factors: the likelihood that the event will occur $(p)$ and its consequences $(V)$ considering both financial and human aspects. A risk therefore is an index of the "expected consequence" of the unwanted event. Two types of losses (financial loss $f$ and human loss $h$ ) are considered in Eq. (9) to quantify the value of $V$ (see [26] for more details) where $c$ represents a factor translating human loss into financial terms. The term $c$ is also known as the Value of a Statistical Life (VSL). Its value depends on a variety of econometric issues which are different from country to country, as well as several factors related

\footnotetext{
${ }^{\dagger}$ This also includes planning, preparation and installation. The initial costs for training, implementation and adaptation are also included in the initial investment.

†We assume that safety investments may include one or multiple safety measures.
} 
to the business sector, the age, the socio-cultural conditions and lifestyles of the persons potentially affected by a ity. For more details about the VSL, readers are referred to [32]. It has been pr that the VSL can significantly influence final decisions about the safety measures to be adopted to prevent major accidents. Therefore, during the decision making process it is recommendable to perform several sensitivity analyses assuming different levels of VSL or, in alternative, assessing various accident scenarios as shown in Section 5

$$
V=f+c \cdot h
$$

Moreover, the risk aversion of a decision-maker towards a high consequence accident scenario can also be considered by using Eq. (10) where $a$ indicates the risk attitude of the decision-maker as shown in Table 3 . For instance, a risk-seeking decisionmaker has a tendency to renounce or postpone in the future investments in safety, because he/she considers the likelihood of a HILP accident to be so low. Together with the DF, the risk factor $a$ can be used as a parameter to balance the risk awareness of the cision-maker as a way to incentive investments in safety. The DF is used to quantify ow many times the benefits from risk reduction are more important than the related costs" according to the decision-maker, while the risk aversion factor measures his/her aversion in accepting severe accidents.

$$
R=p \cdot V^{a}
$$

Table 3: Attitude of the decision-maker towards risk

\begin{tabular}{ll}
\hline$a$ & Description \\
\hline$<1$ & Risk-seeking attitude of decision-maker \\
1 & Risk-neutral attitude of decision-maker \\
$>1$ & Risk-averse attitude of decision-maker \\
\hline
\end{tabular}

Assuming that a safety investment, whose effectiveness is represented by the letter $e$, is adopted, then the risk of an accident can be decreased. In fact, the probability of an accident that might trigger consequences estimated to be equal to $V$, can be lowered due to the safety investment as in the following formula:

$$
R_{\text {with-measure }}=(1-e) \cdot p \cdot V^{a}
$$

Supposing that no safety investment is implemented to prevent a potential accident, its expected risk is measured by Eq. (10). Therefore, the profit of having a safety measure can be quantified as the marginal savings that can be obtained compared to a "no investment" basic case. More specifically, the marginal savings are represented by the avoided expected losses in case of accident due to a lower overall risk. In Eq. (12) the marginal gain is shown.

$$
R_{\text {no-measure }}-R_{\text {with-measure }}=p \cdot V^{a}-(1-e) \cdot p \cdot V^{a}=e \cdot p \cdot V^{a}
$$


Finally, assuming that the safety investment allows to decrease the risk of accidents during a fixed time horizon whose length is $n$, the total effect of the safety measure on the risk reduction can be estimated by multiplying the probability $p$ by $n^{\dagger}$ As a result the potential benefits quantified in the year 0 assume the form reported in Eq. (13).

$$
B_{0}=e \cdot p \cdot n \cdot V^{a}
$$

In light of this, the term $B_{t}$ in Eq. (2) assumes the following value:

$$
B_{t}= \begin{cases}e \cdot p \cdot n \cdot V^{a}, & \text { if } t=0 \\ 0, & \text { otherwise }\end{cases}
$$

\section{Decision model}

During the Risk assessment phase, risk experts should analyze the features of a system that might potentially be affected by a HILP accident. Accident types should be investigated to assess their possible consequences.

These consequences can be estimated from a financial point of view, as described before. The probability of the accident scenario represents another parameter to be estimated.

From this information, a quantification of the expected risk associated to the accident scenario is determined. In Figure 1, we propose a decision model that can be followed within an organization to assess, evaluate and decide about a safety investment regarding an accident scenario.

As can be seen in Figure 1, this risk assessment approach serves as an input for both a technical and a financial assessment. These phases can be executed in parallel, based on the findings of the previous step. The technical assessment is focused on the definition of the most suitable safety investments, based on the threats that might affect the system. A list of investments available on the market is produced and for each of them some basic features are determined (e.g. installation cost, maintenance, effectiveness, duration). Moreover, some of the available investments might not be compatible, from a technical point of view, with the system to be protected. For this reason the incompatible measures should be discarded from the following steps of the analysis.

Furthermore, the goal of the Financial assessment is to define the safety budget that might discard some of the safety investments being too expensive. Moreover, some of the parameters used to estimate the benefit of safety investments need to be defined, such as the discount rate and the time horizon to analyze the investment. Evidently, the time horizon should be compatible with the duration of the safety investment being considered.

For every feasible safety investment, an evaluation is subsequently performed to analyze its financial impact and to determine the DF which makes the safety investment

\footnotetext{
$\dagger$ In other words $p$ is the expected annual probability (or frequency) of an accident. By multiplying $p$ by $n$ we implicitly assume that $p$ remains constant every year.
} 


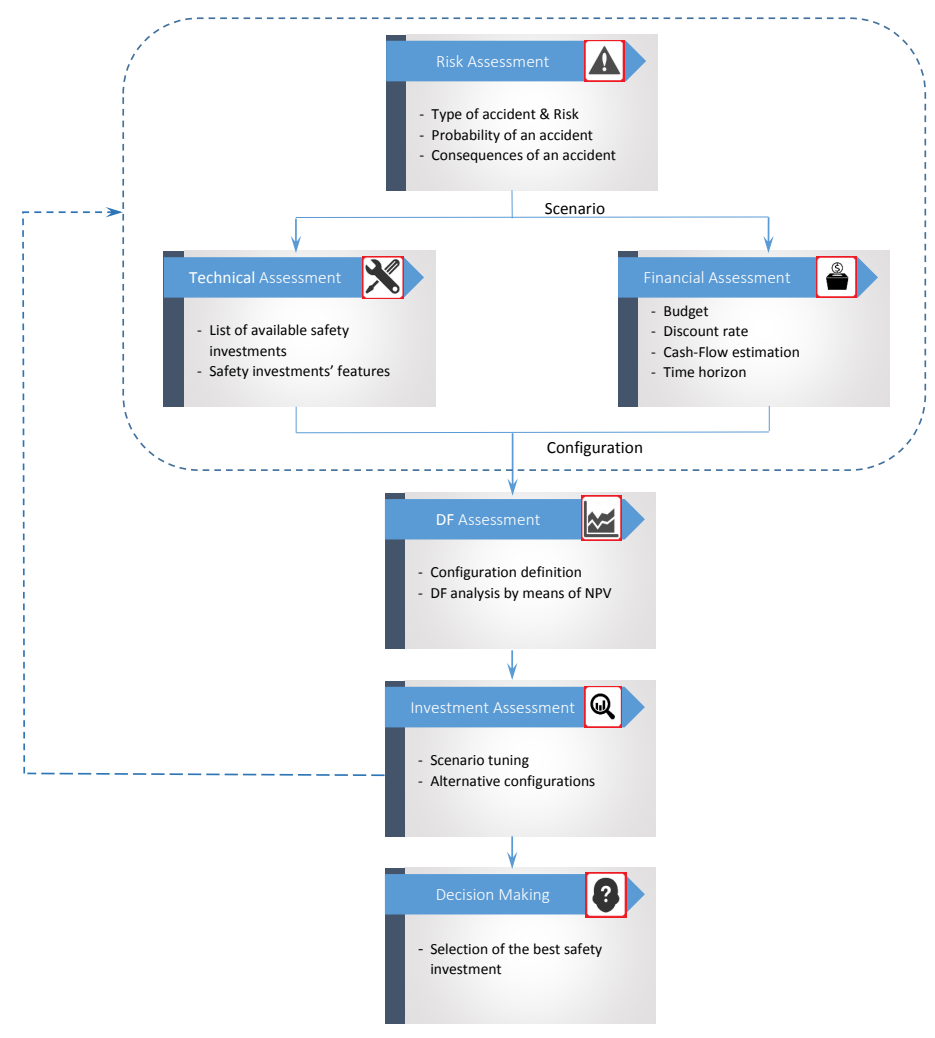

Figure 1: general scheme of the decision model

profitable. A specific configuration might be required by the financial and technical assessment phase to evaluate the investment. This configuration provides specific information in terms of features of the selected safety investment, time horizon, discount rate etc., which can be used to evaluate the investment. Moreover, several scenarios can be analyzed by carrying out a sensitivity analysis whereby the consequences and probabilities of accident scenarios are used as test parameters.

Therefore, the main goal of the Investment assessment is to evaluate the robustness of the choice under different scenarios and assumptions. More specifically, different configurations might be tested to explore how the DF associated to the safety measure under investigation is affected if one of the test parameters changes. Sometimes, the financial and the technical assessment needs to be reiterated to realign the technical and the financial elements included in the decision model.

For each measure the proposed process can be repeated. In addition, other investments can be analyzed and compared with each other. In some cases, especially for HILP accidents, where there is no consensus between risk experts, alternative scenarios, presenting e.g. higher or lower accident probabilities, might be considered. Afterwards, the whole decision approach is repeated to assess the impact on the safety investments to be selected. The final step is represented by the Decision making in 
which alternative investments are evaluated on the basis of elements such as the DF for which the NPV is equal to zero. Supposing to have alternative investment options requiring the same initial investments, the one presenting the lower DF is to be preferred from a financial point of view. Different simulations can be carried out such as comparing measures given the same scenario (consequence and likelihood of an accident) and/or configuration such as the time horizon.

\section{Simulation on illustrative case studies}

In this section some analyses are carried out to simulate the decision process that can be followed while evaluating some real-life safety investments in realistic scenarios. Section 5.1 describes the features of the safety measures and the values of the main technical and financial parameters which are considered in some illustrative case studies, while Section 5.2 provides some recommendations and explanations for the outcomes of the simulation exercise.

\subsection{Input information}

The decision model described in Section 4 has been widely tested on realistic data representing some of most used safety measures within chemical plants to prevent and/or mitigate domino effects (see [16] for more details). More specifically, the safety measures listed in Table 4 have been used to validate the decision model described before. The costs associated to these measures include all the initial costs (e.g. selection and design costs, training and organizational changes), the installation costs (e.g. material and purchase costs, start-up and equipment costs) and other costs related to the safety of a large chemical plant (and all its critical installations) located in an industrial area subject to domino effect accidents.

Table 4: Features of the safety investments

\begin{tabular}{llll}
\hline id & Description & $M$ (in million euro) & $e$ \\
\hline 1 & Concrete wall surrounding tank of 25 meter + sprinkler without additional foam & 15 & $95 \%$ \\
2 & Automatic sprinkler installation with additional foam & 10 & $93 \%$ \\
3 & Automatic sprinkler installation without additional foam & 8 & $90 \%$ \\
4 & Deluge system (water spray system opened as signaled by a fire alarm system) & 4 & $86 \%$ \\
5 & Fire-resistant coating & 2 & $81 \%$ \\
\hline
\end{tabular}

A full factorial experiment has been carried out, testing the effects of the selected safety measures on different scenarios (summarized in Table 5) and technical and financial configurations (reported in Table 6). 
Table 5: Possible scenarios after the risk assessment

\begin{tabular}{l}
\hline Type of accident \\
Vapor Cloud Explosions and Fires, BLEVE without chemical reactions, BLEVE with chemical reactions \\
\hline Maximum estimated damages potentially triggered by the accident $(V)$ in million euro \\
$0.5,1,2,5,10$ \\
\hline Probability of the accident $(p)$ \\
$10^{-4}, 10^{-5}, 10^{-6}, 10^{-7}, 10^{-8}$ \\
\hline Risk aversion $(a)$ \\
$1.3,1.35,1.4,1.45,1.5,1.55,1.6$
\end{tabular}

Table 6: Tecnical and finantial parameters

Discount rate $(i)$

\begin{tabular}{l}
$1 \%, 2 \%, 3 \%$ \\
\hline Yearly recurrence cost $(m)$ \\
$1 \%, 3 \%, 5 \%, 10 \%$, \\
\hline Time horizon $(n)$ \\
$5,10,15,25$ \\
\hline
\end{tabular}

\subsection{Results and recommendations}

A sensitivity assessment was performed using both the outcomes of the risk assessment and the test parameters (including the consequences and the probabilities of a potential accident) to evaluate possible safety investments.

The analysis is carried out by fixing some of the parameters decided during the risk, financial and technical assessments and analyzing the influence of the remaining parameters, which are free to be changed) on the DF. In the remainder of the paper, if not expressly mentioned, the basic parameters setting summarized in Table 7 has been adopted to produce the graphs.

\begin{tabular}{lr}
\hline Parameter & Value \\
\hline Risk attitude $(a)$ & 1.45 \\
Yearly recurring cost $(m)$ & $3 \%$ \\
Time horizon $(n)$ & 25 \\
Discount rate $(i)$ & $2 \%$ \\
Probability of accident $(p)$ & $10^{-5}$ \\
\hline
\end{tabular}

Table 7: Basic parameters setting used in the simulation

In Figure 2, five safety investments are assessed based on the calculated DF whereby the NPV equals zero and considering three different accidents scenarios, and hence three hypothetical benefits (that is 2,5 and 10 million euro). In fact, in many real-life 
decisions, in order to deal with the uncertainties associated to the value of $V$ (the consequences of a potential accident), decision-makers should consider several accident scenarios, hence assuming different sensitivities, under which assessing alternative safety measures. From now on the value of the DF which makes the NPV equal to zero is denoted by $\mathrm{DF}^{0}$ to avoid confusion and simplify the reading.

As expected, measure 5 (i.e. "fire resistant coating" see Table 5) presents a lower $\mathrm{DF}^{0}$ because the investment it requires is lower compared to the other measures. Therefore, from a point of view of an investor, the benefits are more proportionate to the costs. With reference to measure 1 a higher $\mathrm{DF}^{0}$ needs to be used to justify the selection of this measure that nevertheless has a higher effectiveness to prevent and mitigate major accidents. As shown in Figure 2, when the potential benefits of preventing major accidents increase, the $\mathrm{DF}^{0}$ values associated to the different safety investments decrease. This result is justifiable from a decision-maker's perspective because investments are more proportional to potential savings.

It should be noted that sometimes cheaper safety investments imply the use of simple safety measures (e.g. measures 4 and 5 in Figure 2) which might not be viable options for major accidents due to technical and safety reasons (e.g. a reduced effectiveness to prevent major accidents). Therefore, these options should be discarded during the risk assessment phase, while considering catastrophic accident scenarios.

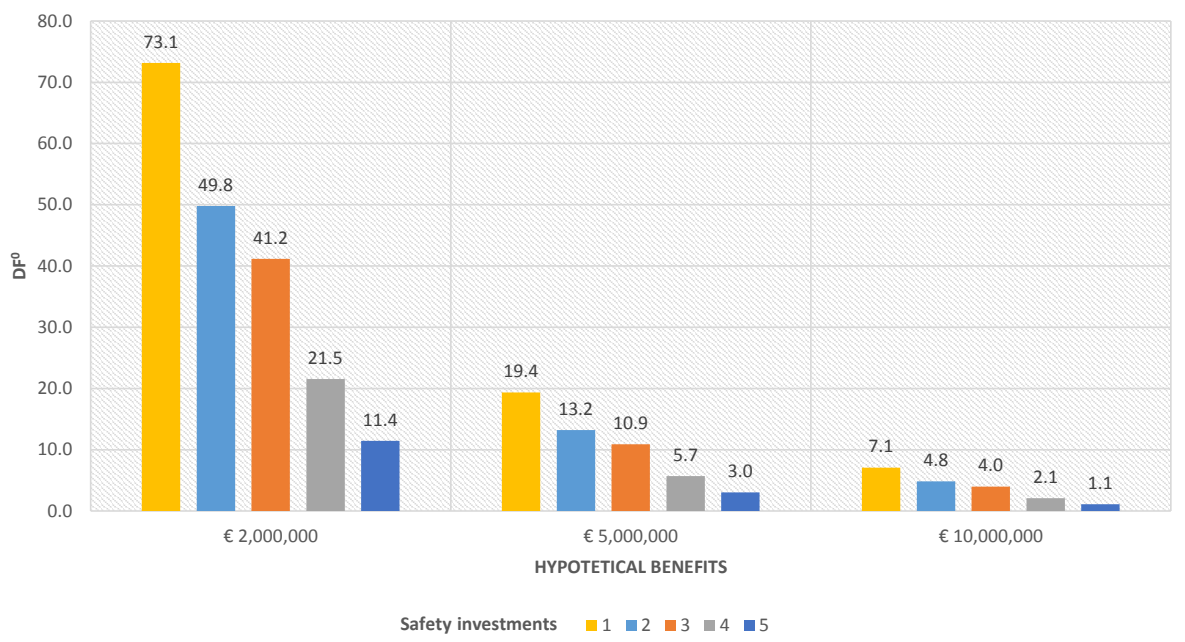

Figure 2: $\mathrm{DF}^{0}$ associated to alternative measures, while evaluating different accident scenarios

\subsubsection{Impact of different accident scenarios on the $D F^{0}$}

After having shortlisted the investments to be analyzed in the decision process, one might consider to focus on a single investment to assess its robustness by studying the relationship between the main features of the safety investments and the $\mathrm{DF}^{0}$. In this way, alternative options can be better compared and assessed using a multidimensional decision-making approach. For example, in Figure 3 a specific investment (in this case option number 3 "Automatic sprinkler installation without additional foam") is 
investigated using the parameter setting in Table 7 Assuming three different accident scenarios (and thus three different values of hypothetical benefits), a simulation on the relationship between the $\mathrm{DF}^{0}$ and the NPV is shown. As expected, the higher the potential benefit, the lower the $\mathrm{DF}^{0}$ required to make the investment profitable.

Analyzing Figure 3 it is possible to see that the safety investment is justifiable from a financial point of view only if the hypothetical benefits are greater than $€ 2$ million. In fact, for minor accident scenarios (in this case hypothetical benefits lower or equal to $€ 2$ million) the total costs of the investment can compensate the potential benefits, only when assuming a disproportion factor greater than 30 . Since disproportion factors greater than 30 are generally considered by investors too high, the associated measures do not represent reasonable investments from an economic perspective and thus they should be ruled out when the accident scenarios, which may affect an organization, are triggering "minor" consequences.

Considering accidents with a significant impact e.g. triggering damages equal to $€ 5$ or $€ 10$ million, the measure presents a zero net present value for values of disproportion factors equal to 10.9 and 4.0 respectively. Since, these values of disproportion factors are below 30, and thus reasonable according to the study of Goose [11], the measure represents a viable investment option to be considered by decision-makers in case of major accidents.

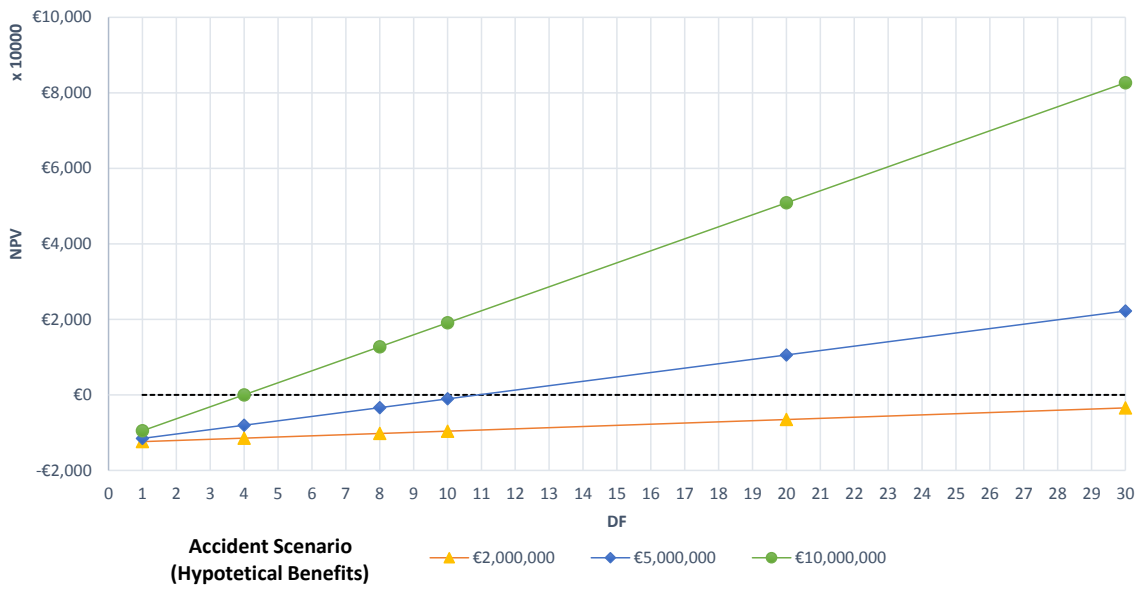

Figure 3: NPV and DF associated to different accident scenarios

In Figure 4 the impact of the probability of a potential accident is assessed. In particular, the higher the probability, the lower the value of the $\mathrm{DF}^{0}$ that is needed to make the investment profitable from a financial point of view. If the probability of the accident is very low, the decision-maker will tend to avoid safety investments since the hypothetical benefits are more uncertain and more disproportionate than costs. For those reasons, a higher disproportion factor is required to stress the importance of safety in case of HILP accidents. 


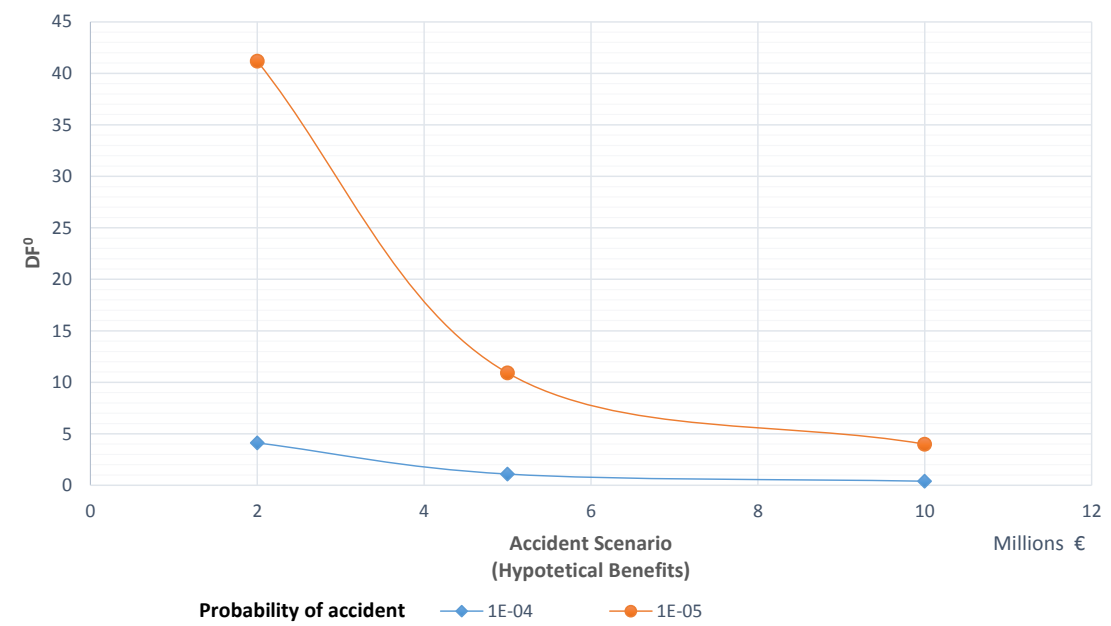

Figure 4: Relationship between the probability fo accident and the $\mathrm{DF}^{0}$ associated to different scenarios

\subsubsection{Impact of technical and financial parameters on the $D F^{0}$}

Focusing on a specific safety investment, the relationships between financial-technical parameters and the $\mathrm{DF}^{0}$ can be further explored.

In Figure 5 the relationship between the yearly recurring costs and the $\mathrm{DF}^{0}$ is shown for a specific safety investment (in this case measure number 2 "Automatic sprinkler installation with additional foam"), while considering hypothetical benefits of $€ 5$ million. The other technical and financial parameters are set to the values reported in Table 7. As expected, the greater the yearly recurring costs, the higher the $\mathrm{DF}^{0}$ due to increased sacrifices in safety required to a firm.

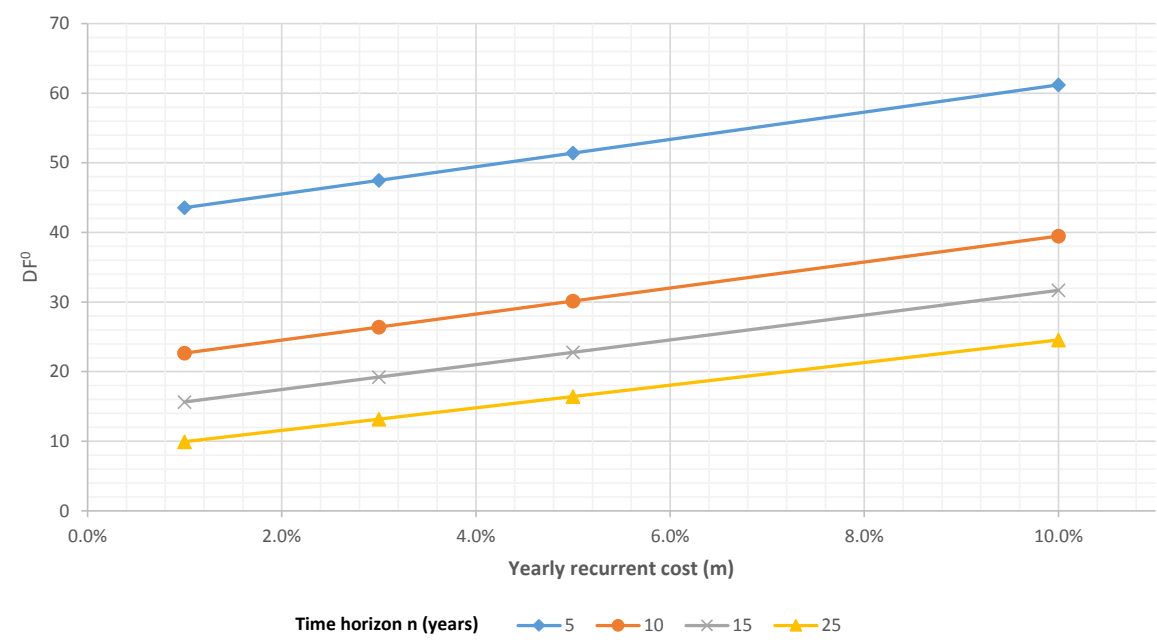

Figure 5: Relationship between the $\mathrm{DF}^{0}$ and the yearly recurring costs for different time horizons 
A simulation considering also different time horizons, in which the safety measure can maintain its effectiveness, has been performed. As shown in Figure 6 the longer the time in which the safety investment can maintain its effectiveness before becoming obsolete, the more attractive the investment is and thus the lower the $\mathrm{DF}^{0}$. In fact, the shorter the time horizon, the higher the $\mathrm{DF}^{0}$ should be to make the investment desirable due to a shorter time period for the return on investment.

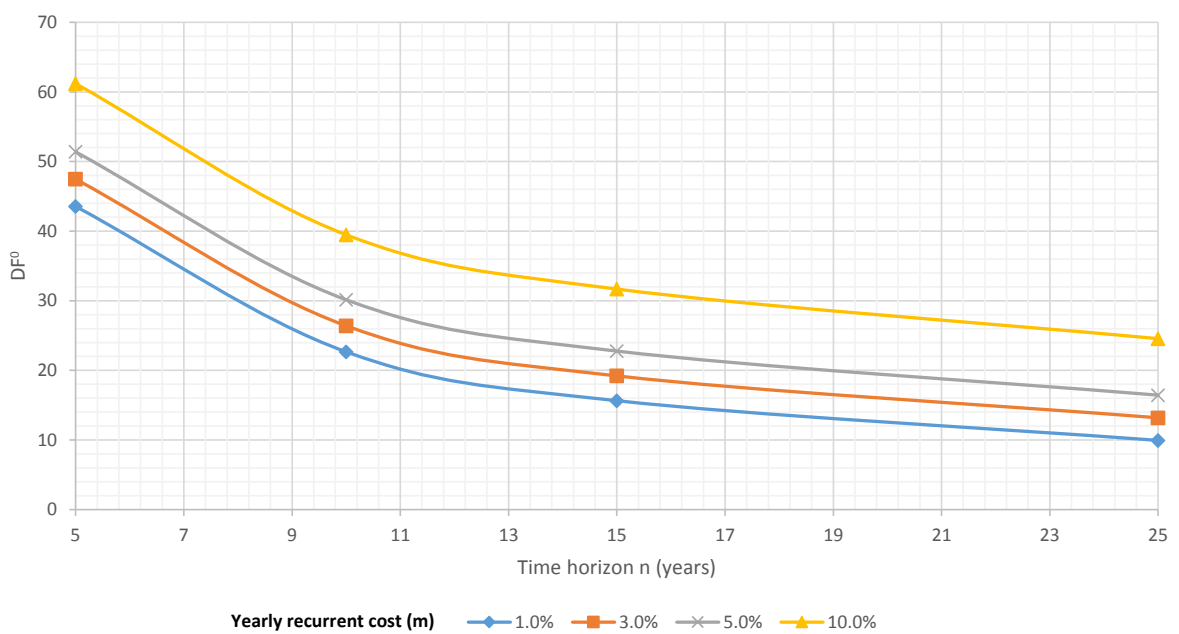

Figure 6: Relationship between the $\mathrm{DF}^{0}$ and the asset life for different value of maintenance cost

Still considering safety investment number 2, using the same accident scenario analyzed before and freezing the remaining technical and financial parameters as in Table 7. Figure 7]illustrates the relationship between the discount factor used to calculate the NPV with the $\mathrm{DF}^{0}$. Figure $7(\mathrm{a})$ shows that the $\mathrm{DF}^{0}$ remains stable for different values of the discount rate given a fixed time horizon. Moreover, the larger the time horizon in which the investment is analyzed, the lower the $\mathrm{DF}^{0}$. As shown in Figure 7(b), the $\mathrm{DF}^{0}$ is not significantly affected by a variation of the discount factor as far as this latter remains below a certain level. However, the $\mathrm{DF}^{0}$ can vary depending on the maintenance cost that is considered. In addition, when the maintenance costs are high, an increase in the discount factor can trigger a decrease of the $\mathrm{DF}^{0}$.

\subsubsection{Impact of the risk aversion factor on the $D F^{0}$}

Finally the relationship between a decision-maker's risk attitude and the $\mathrm{DF}^{0}$ can be assessed. Figure 8 represents the evolution of the $\mathrm{DF}^{0}$ when the risk aversion factor assumes different values for two different safety investments. Moreover, the lower the potential benefit, the lower the $\mathrm{DF}^{0}$ that would be used by the decision-maker to evaluate the investment (see Figure 8(a) and Figure 8(b)].

As expected, the lower the investment required by a safety measures, the lower the $\mathrm{DF}^{0}$ that is needed to make the safety costs proportional to the potential benefits. When the value of $a$ increases, it means that the decision-maker is more risk averse and therefore he will be more inclined towards safety investments. Since both the discount 


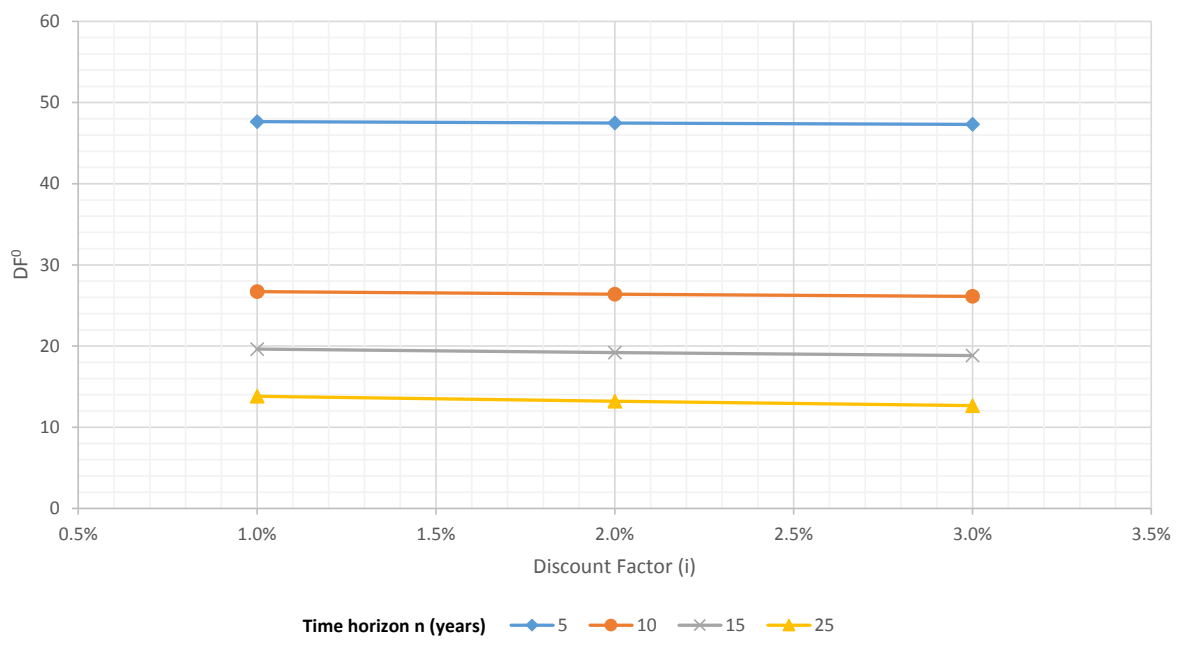

(a) Relationship between the $\mathrm{DF}^{0}$ and the discount factor for different time horizons

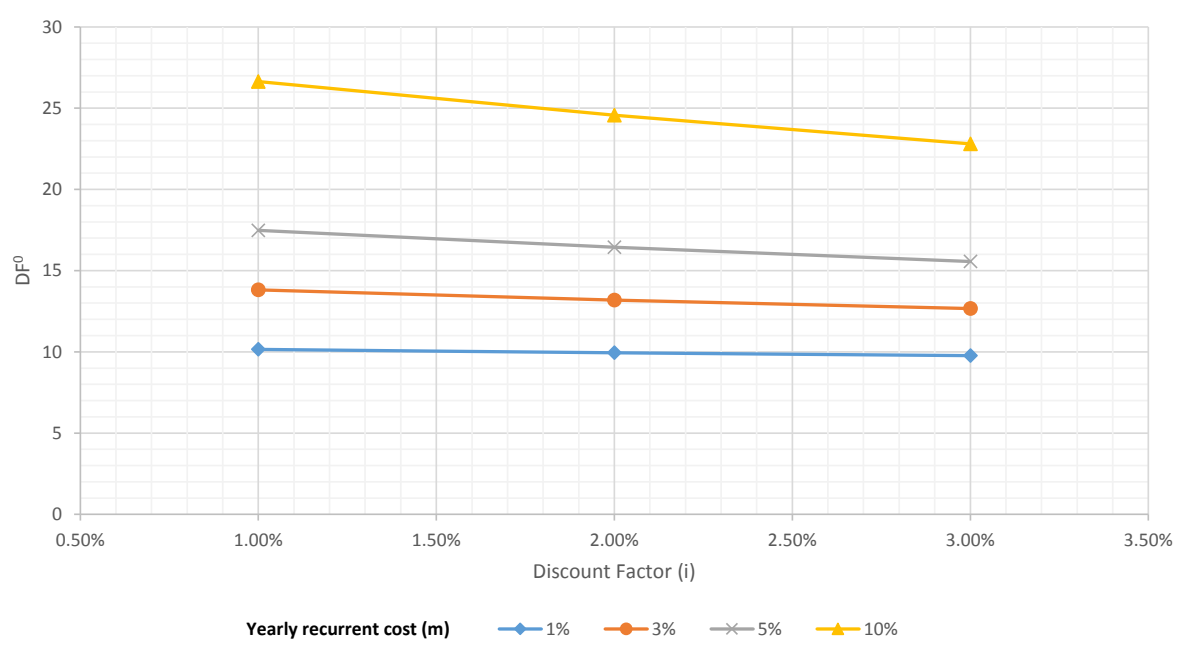

(b) Relationship between the $\mathrm{DF}^{0}$ and the discount factor for different yearly recurrent costs

Figure 7: Relationship between the $\mathrm{DF}^{0}$ and the discount factor

factor and the risk aversion factor can be used as a bias in favor of safety, when the value of $a$ increases there is a reduced need to use the $\mathrm{DF}^{0}$ as an incentive for investments in safety. 


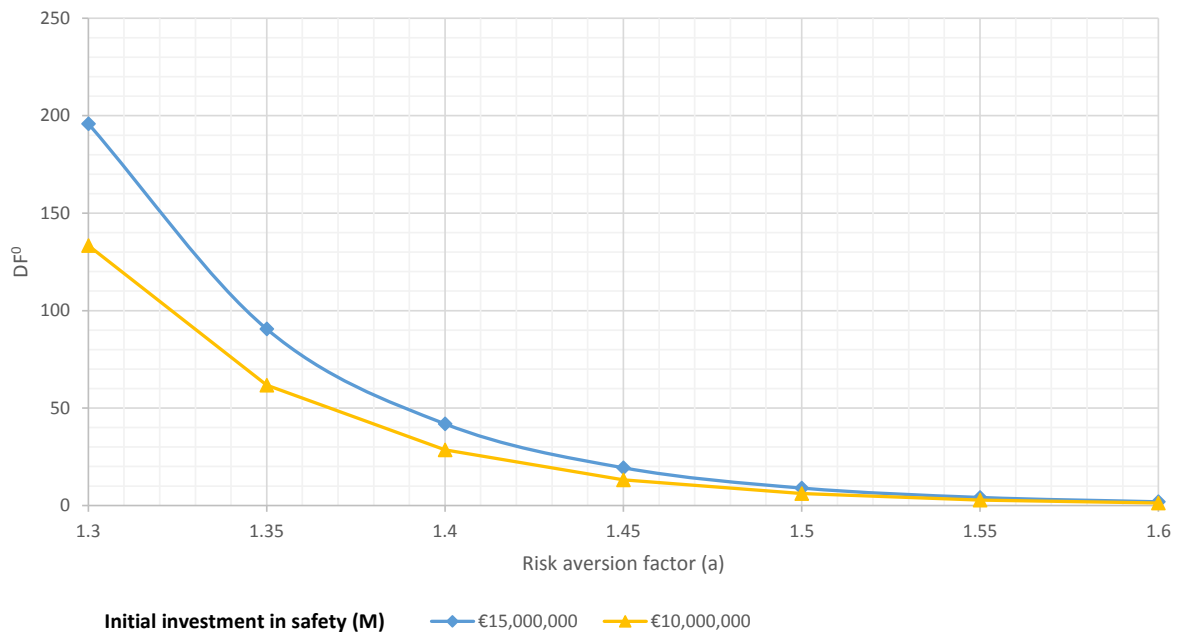

(a) Potential benefits equal to $€ 2$ million

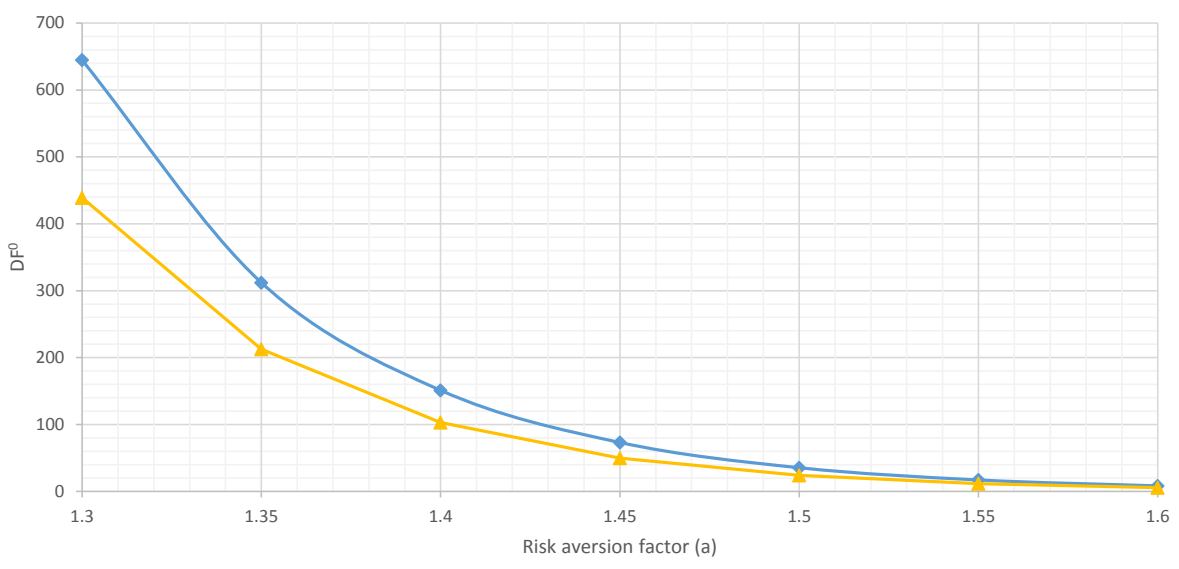

Initial investment in safety (M) $\rightarrow \multimap € 15,000,000 \quad \rightarrow-€ 10,000,000$

(b) Potential benefits equal to $€ 5$ million

Figure 8: Relationship between the DF and the risk factor for different accident scenario]

\section{Conclusions and future work}

In this paper we presented a structured approach to carry out a quantitative costbenefit analysis for safety investments aimed at HILP risks. A disproportion factor has been used to make safety costs comparable with the expected hypothetical benefits that such investments might trigger due to accident prevention. A decision model is presented showing a possible approach to assess and evaluate mid-long term safety decisions considering technical, financial and risk management aspects.

The goal of the methodology is to define the level of disproportion factor which makes the investment profitable from a financial point of view. The approach can be 
used to classify and rank alternative investments, rather than defining ideal levels of disproportion factor which makes the safety investment profitable. These latter may depend on several factors such as the type of industry, the type of accidents, the reputation of the organization, legal framework etc. However, to support the decision process and the selection of the ideal safety investment to implement one should consider that reasonable values of disproportion factor might lay in the range $[1,30]$. In light of this, safety investments with $\mathrm{DF}^{0}$ outside of this ranges are to be discarded. For this reason the methodology presented in this paper can be used to shortlist and rank alternative options and provide quantitative rationales for decision-makers to justify safety investments. Similarly to other financial indicators and ratios (e.g. NPV, Internal Rate of Return), in which recommendations are provided to support decision-making, here we suggest that assuming all safety investments requiring the same initial investment, the alternative with the lowest $\mathrm{DF}^{0}$ falling in an interval which is considered reasonable would be considered the best option from a financial point of view. Moreover, an organization should, in theory, undertake all safety investments with $\mathrm{DF}^{0}$ lower or equal to 1 when supported by available safety budgets.

A simulation has been performed, analyzing the relationships between the main financial and technical parameters and the disproportion factor. This information can be used by decision-makers to compare and analyze alternative safety investments which can be effectively adopted to prevent and mitigate specific accident scenarios.

To conclude, the quantitative assessment of safety investments by using the disproportion factor and the NPV represents a complex topic. Despite the sensitivity simulation approach proposed in this paper being quite flexible and easily adjustable by the decision-maker, it represents a first attempt to introduce this topic in the literature. Without any doubt the subject is highly relevant for the industry and the policy makers and it deserves further study. Some of the issues that are worth to address within future work refer to: (a) the accuracy of the data used in the model (i.e. How to include uncertainty in the model? How to measure the effectiveness of a safety measure? How the benefits from the community can be estimated?); (b) the methodological aspects (i.e. What other models, different from the NPV, can be used?) (c) standards and best practices based on the most reasonable values of the disproportion factor to be used for different type of accidents (and its features such as risk, probability, consequences), type of companies/industry and categories of safety measures.

While the first two aspects can be addressed from a more theoretical point of view, the last item represents a challenging task due to a lack of statistical data based on real cases and current practices from the industry. A better investigation of all these aspects may certainly improve the overall safety level in the industry, creating at the same time the conditions for governments or public institutions, to define enhanced safety policies. 


\section{References}

[1] D. Andreoni. The cost of occupational accidents and diseases. Number 54. Intl Labour Organisation, 1986.

[2] L. J. Asquith. Edwards v. national coal board. All England Law Reports, 1:747, 1949.

[3] P. Baybutt. The ALARP principle in process safety. Process Safety Progress, 33(1):36-40, 2014.

[4] H. F. Campbell and R. P. Brown. Benefit-cost analysis: financial and economic appraisal using spreadsheets. Cambridge University Press, 2003.

[5] CCPS (Center for Chemical Process Safety). Guidelines for Chemical Transportation Safety, Security, and Risk Management. John Wiley and Sons, Hoboken, New Jersey, 2008.

[6] P. Dorman. The economics of safety, health, and well-being at work: an overview. ILO Geneva, 2000.

[7] ENISA. Introduction to Return on Security Investment: Helping CERTs assessing the cost of (lack of) security. European Network and Information Security Agency, Heraklion, Greece, 2012.

[8] W. R. Flores, T. Sommestad, H. Holm, and M. Ekstedt. Assessing future value of investments in security-related it governance control objectives-surveying it professionals. In ICIME 2011-Proceedings of the 2nd International Conference on Information Management and Evaluation: ICIME 2011 Ryerson University, Toronto, Canada, 27-28 April 2011, page 393. Academic Conferences Limited, 2011.

[9] E. F. for the Improvement of Living, W. Conditions, et al. Abstracts from the workshop on occupational health strategies. third european conference on monitoring the working environment. Technical report, 1997.

[10] S. French, T. Bedford, and E. Atherton. Supporting ALARP decision making by cost benefit analysis and multiattribute utility theory. Journal of Risk Research, 8(3):207-223, 2005 .

[11] M. Goose. Gross disproportion, step by step-a possible approach to evaluating additional measures at COMAH sites. In Institution of chemical engineers symposium series, volume 151, page 952. Institution of Chemical Engineers; 1999, 2006.

[12] L. A. Gordon and M. P. Loeb. The economics of information security investment. ACM Transactions on Information and System Security (TISSEC), 5(4):438-457, 2002.

[13] A. R. Hale, B. Heming, J. Carthey, and B. Kirwan. Modelling of safety management systems. Safety Science, 26(1):121-140, 1997.

[14] G. B. Health and S. Executive. Quantified Risk Assessment: Its Input to Decision Making. HM Stationery Office, 1989.

[15] HSE Books. Reducing risks protecting people: HSEs decision-making process, 2001.

[16] J. Janssens, L. Talarico, G. Reniers, and K. Sörensen. A decision model to allocate protective safety barriers and mitigate domino effects. Reliability Engineering \& System Safety, 143:44-52, 2015. 
[17] P. Miller, D. Whynes, and A. Reid. An economic evaluation of occupational health. Occupational Medicine, 50(3):159-163, 2000.

[18] P. Miller, P. Rossiter, and D. Nuttall. Demonstrating the economic value of occupational health services. Occupational Medicine, 52(8):477-483, 2002.

[19] J. Mossink. Understanding and performing economic assessments at the company level. 2002.

[20] G. Reniers and T. Brijs. Major accident management in the process industry: An expert tool called cesma for intelligent allocation of prevention investments. Process Safety and Environmental Protection, 92(6):779-788, 2014.

[21] A. Robens. Safety and health at work: report of the Committee, 1970-72, volume 1. HM Stationery Off., 1972.

[22] M. D. Rogers. The european commission's white paper strategy for a future chemicals policy: a review. Risk Analysis, 23(2):381-388, 2003.

[23] S. Schneider. The economics of health and safety in construction. Laborers' Health and Safety Fund of North America, pages 1-12, 2006.

[24] B. Schneier. Security ROI: Fact or fiction. CSO Magazine (September 2008), 2008.

[25] C. Smallman and G. John. British directors perspectives on the impact of health and safety on corporate performance. Safety science, 38(3):227-239, 2001.

[26] L. Talarico, G. Reniers, K. Sörensen, and J. Springael. Mistral: A game-theoretical model to allocate security measures in a multi-modal chemical transportation network with adaptive adversaries. Reliability Engineering \& System Safety, 138:105-114, 2015.

[27] A. Targoutzidis, T. Koukoulaki, E. Schmitz-Felten, K. Kuhl, K. Oude Hengel, E. Rijken, R. Kluser, et al. The business case for safety and health at work: cost-benefit analyses of interventions in small and medium-sized enterprises. European Agendy for Safety and Health at Work, Publications Office of the European Union, Luxembourg, 2014.

[28] P. Thomas and R. Jones. Extending the j-value framework for safety analysis to include the environmental costs of a large accident. Process Safety and Environmental Protection, 88 (5):297-317, 2010.

[29] E. Tompa, R. Dolinschi, C. de Oliveira, and E. Irvin. A systematic review of occupational health and safety interventions with economic analyses. Journal of Occupational and Environmental Medicine, 51(9):1004-1023, 2009.

[30] V. Trbojevic. Risk criteria in EU. Risk, 10(5), 2005.

[31] J. Verbeek, M. Pulliainen, and E. Kankaanpää. A systematic review of occupational safety and health business cases. Scandinavian journal of work, Environment \& Health, pages 403-412, 2009.

[32] W. Viscusi and J. Aldy. The value of a statistical life: A critical review of market estimates throughout the world. Journal of Risk and Uncertainty, 27(1):5-76, 2003.

[33] J. Willemson. On the Gordon \& Loeb model for information security investment. In WEIS, 2006. 